\title{
Editorial
}

\section{Applications of the Holographic Duality to Strongly Coupled Quantum Systems}

\author{
Li Li, ${ }^{1}$ Hai-Qing Zhang, ${ }^{2}$ Song He, ${ }^{3}$ Zhang-Yu Nie, ${ }^{4}$ Yun-Long Zhang, ${ }^{5}$ and Bum-Hoon Lee ${ }^{6}$ \\ ${ }^{1}$ Lehigh University, Bethlehem, PA, USA \\ ${ }^{2}$ Utrecht University, Utrecht, Netherlands \\ ${ }^{3}$ Max Planck Institute for Gravitational Physics, Golm, Germany \\ ${ }^{4}$ Kunming University of Science and Technology, Kunming, Yunnan, China \\ ${ }^{5}$ Asia Pacific Center for Theoretical Physics, Pohang, Republic of Korea \\ ${ }^{6}$ Sogang University, Seoul, Republic of Korea \\ Correspondence should be addressed to Li Li; lil416@lehigh.edu
}

Received 28 November 2017; Accepted 28 November 2017; Published 14 December 2017

Copyright (C) $2017 \mathrm{Li} \mathrm{Li}$ et al. This is an open access article distributed under the Creative Commons Attribution License, which permits unrestricted use, distribution, and reproduction in any medium, provided the original work is properly cited. The publication of this article was funded by SCOAP ${ }^{3}$.

The holographic duality represents a major advance in our understanding of string theory and quantum gravity and also provides a powerful toolkit for studying strongly coupled quantum field theories. Thanks to this duality, challenging questions about dynamics in quantum phases of matter in strong coupling regime can be mapped to processes in theories of gravity which are tractable. It was proven to be very useful in describing unconventional physics in many fields, such as gauge theory, condensed matter, information theory, and systems described by hydrodynamics.

The selected contributions to this special issue are aimed at bringing together some of the latest developments in this field. The special issue covers several interesting topics, including novel black hole solutions, holographic QCD, entanglement entropy, and the construction of the bulk operators. The accepted articles are highlighted as follows.

The paper titled "Heavy Quark Potential with Hyperscaling Violation" by Z. Zhang et al. investigates the behavior of the heavy quark potential in the Lifshitz backgrounds with hyperscaling violation. It shows how the potential changes in the presence of the nonrelativistic parameters. The effect of a constant electric field on the potential is also investigated.

The paper titled "Holographic van der Waals Phase Transition for a Hairy Black Hole" by X.-X. Zeng and Y.-W. Han is devoted to studying the van der Waals phase transition in a hairy black hole by holography. The two-point correlation function is employed to probe the phase structure of the black hole, and some interesting behaviors are found in the temperature-thermal entropy plane.

The paper titled "Entropic Destruction of Heavy Quarkonium from a Deformed $\mathrm{AdS}_{5}$ Model" by Z. Zhang et al. discusses the effects of the deformation parameter on the interdistance, the entropic force, and the quarkonium dissociation.

The paper by X.-M. Liu et al. is titled "Van der Waals-Like Phase Transition from Holographic Entanglement Entropy in Lorentz Breaking Massive Gravity." The authors use the entanglement entropy to probe the phase transition of AdS black holes in Lorentz breaking massive gravity. It suggests some potential relation between the entanglement entropy and the Bekenstein-Hawking entropy.

In the paper titled "Bulk Local Operators, Conformal Descendants, and Radial Quantization," Z.-L. Wang and Y. Yan establish a construction of the bulk local operators in AdS by considering CFT at finite energy scale. The authors also discuss a possible way of generalizing the construction beyond pure AdS.

The paper titled "Note on Relation between Bottom-Up Holographic Models and Large- $N_{c}$ QCD" by S. S. Afonin 
discusses a derivation of holographic actions quadratic in fields from general properties of the large- $N_{c}$ limit in QCD.

The paper titled "AdS Black Hole with Phantom Scalar Field" by L. Zhang et al. deals with an AdS black hole solution with Ricci flat horizon in Einstein-phantom scalar theory. After discussing the thermodynamics of the phantom black hole, the authors present the behavior of the entanglement entropy for a strip geometry.

In the paper titled "Reentrant Phase Transitions and Triple Points of Topological AdS Black Holes in Born-InfeldMassive Gravity" by M. Zhang et al., the authors study the critical behaviors of topological AdS black holes in the presence of Born-Infeld nonlinear electrodynamics in massive gravity. By considering the cosmological constant as a dynamical pressure of the system, some interesting phase structures are uncovered.

In summary, the holographic duality provides an invaluable source of physical intuition as well as computational power to deal with strongly coupled problems. Constructing, exploring, and categorizing the diverse states of matter through holography are a prominent theme in this special issue. We hope that this special issue will enrich readers with new investigations on the current related research areas and stimulate further studies.

$\mathrm{Li} \mathrm{Li}$

Hai-Qing Zhang Song He

Zhang-Yu Nie

Yun-Long Zhang

Bum-Hoon Lee 

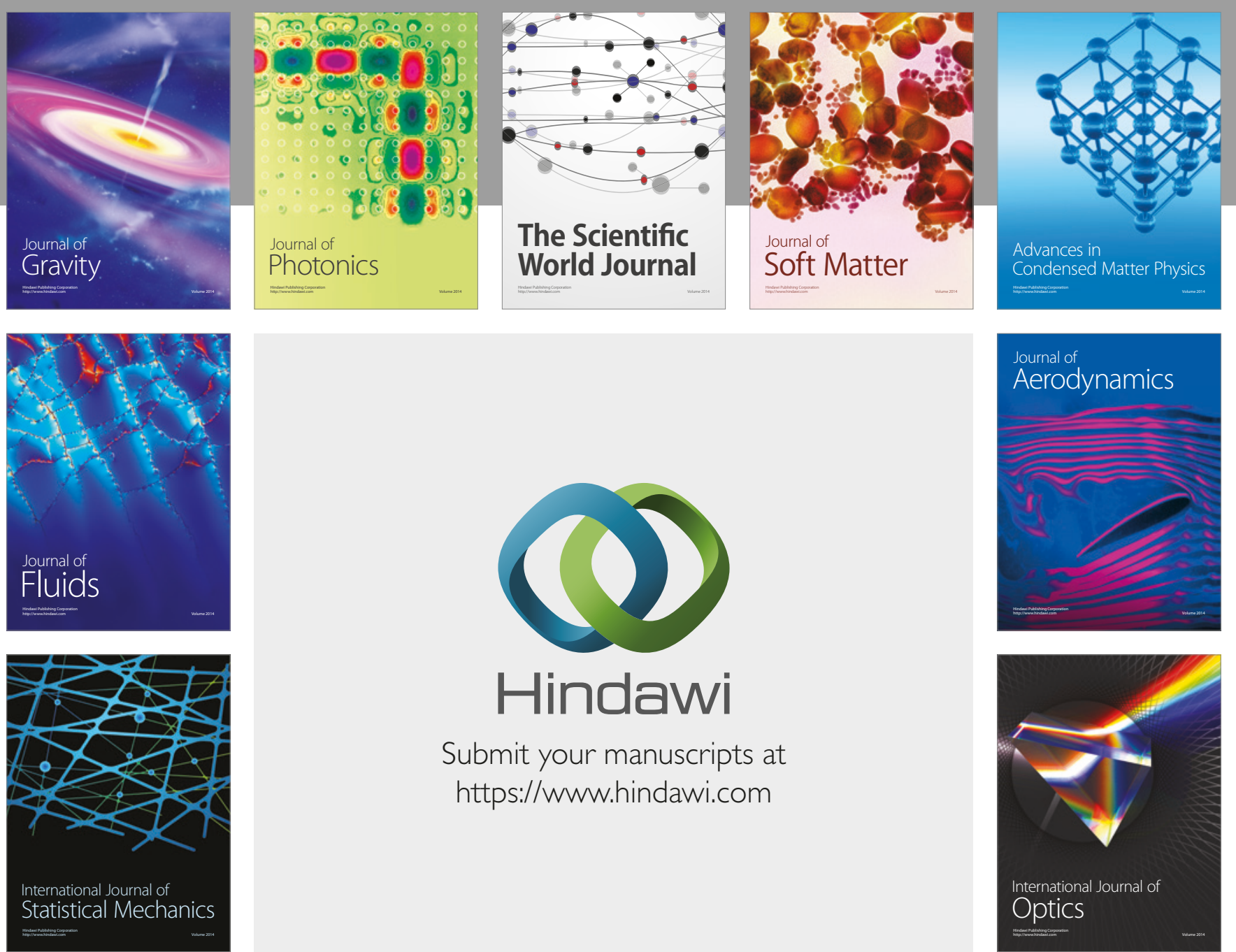

Submit your manuscripts at

https://www.hindawi.com
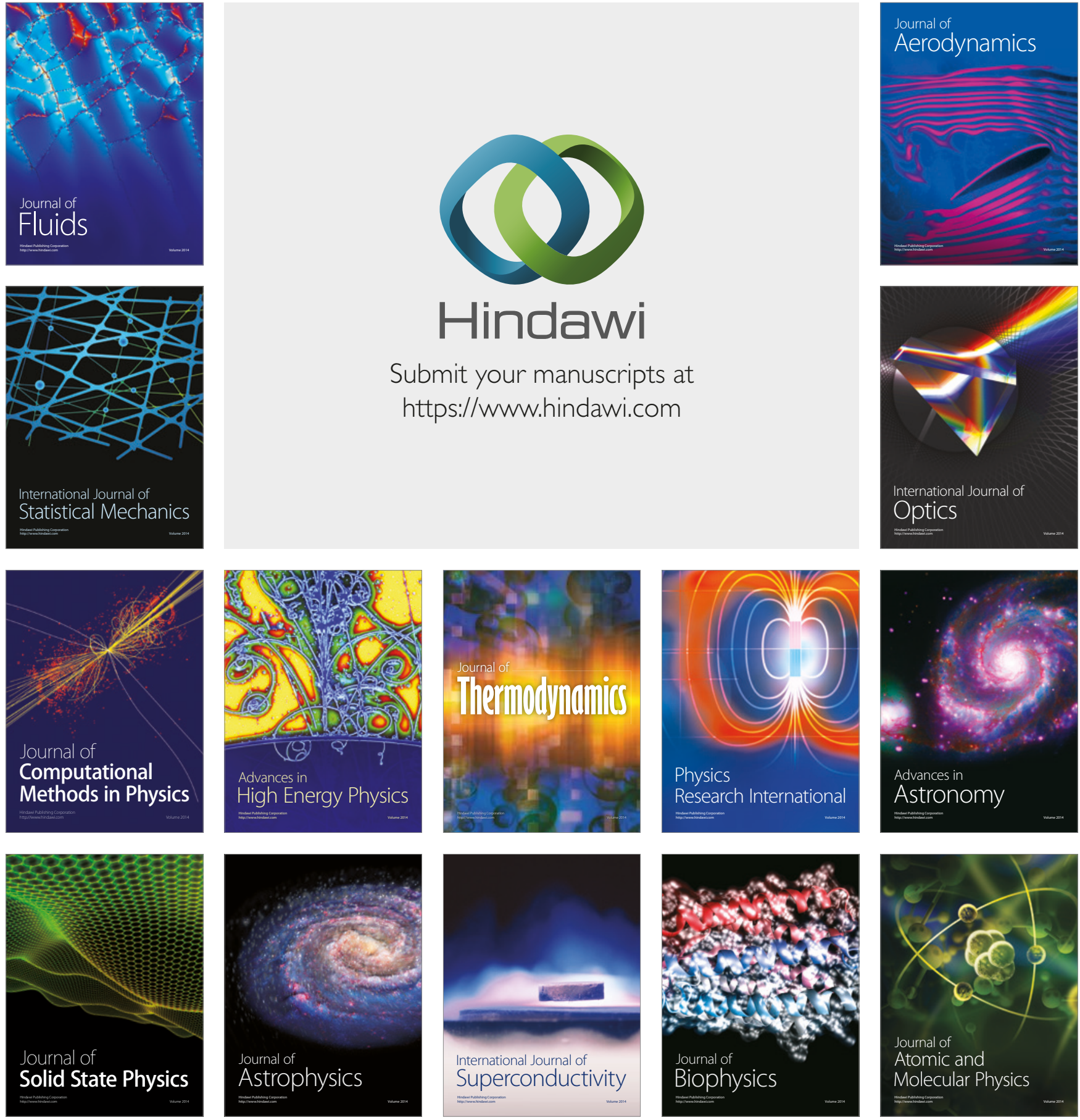